\title{
Formation and early evolution of massive black holes
}

\author{
Piero Madau \\ Department of Astronomy and Astrophysics, University of California, \\ Santa Cruz, CA 95064, USA \\ email: pmadau@ucolick.org
}

\begin{abstract}
The astrophysical processes that led to the formation of the first seed black holes and to their growth into the supermassive variety that powers bright quasars at $z \sim 6$ are poorly understood. In standard $\Lambda \mathrm{CDM}$ hierarchical cosmologies, the earliest massive holes (MBHs) likely formed at redshift $z \gtrsim 15$ at the centers of low-mass $\left(M \gtrsim 5 \times 10^{5} \mathrm{M}_{\odot}\right)$ dark matter "minihalos", and produced hard radiation by accretion. FUV/X-ray photons from such "miniquasars" may have permeated the universe more uniformly than EUV radiation, reduced gas clumping, and changed the chemistry of primordial gas. The role of accreting seed black holes in determining the thermal and ionization state of the intergalactic medium depends on the amount of cold and dense gas that forms and gets retained in protogalaxies after the formation of the first stars. The highest resolution N-body simulation to date of Galactic substructure shows that subhalos below the atomic cooling mass were very inefficient at forming stars.
\end{abstract}

Keywords. Black hole physics - cosmology: theory - galaxies: formation - intergalactic medium - Galaxy: halo

\section{Introduction}

The strong link observed between the masses of MBHs at the center of most galaxies and the gravitational potential wells that host them (Ferrarese \& Merritt 2000; Gebhardt et al. 2000) suggests a fundamental mechanism for assembling black holes and forming spheroids in galaxy halos. In popular cold dark matter (CDM) hierarchical cosmologies, small-mass subgalactic systems form first to merge later into larger and larger structures. According to these theories, some time beyond a redshift of 15 or so, the gas within halos with virial temperatures $T_{\text {vir }} \gtrsim 10^{4} \mathrm{~K}-$ or, equivalently, with masses $M \gtrsim 10^{8}[(1+z) / 10]^{-3 / 2} \mathrm{M}_{\odot}-$ cooled rapidly due to the excitation of hydrogen Ly $\alpha$ and fragmented. Massive stars formed with some initial mass function (IMF) and synthesized heavy elements. Such early stellar systems, aided perhaps by a population of accreting black holes in their nuclei, generated the ultraviolet radiation and mechanical energy that reheated and reionized the cosmos. It is widely believed that collisional excitation of molecular hydrogen may have allowed gas in even smaller systems - virial temperatures of a thousand $\mathrm{K}$, corresponding to masses around $5 \times 10^{5}[(1+z) / 10]^{-3 / 2} \mathrm{M}_{\odot}-$ to cool and form stars at even earlier times (e.g. Tegmark et al. 1997).

The first generation of seed MBHs must have formed in subgalactic units far up in the merger hierarchy, well before the bulk of the stars observed today: this is in order to have had sufficient time to build up via gas accretion a mass of several $\times 10^{9} \mathrm{M}_{\odot}$ by $z=6.4$, the redshift of the most distant quasars discovered in the Sloan Digital Sky Survey (SDSS) (Figure 1; Volonteri \& Rees 2005). In hierarchical cosmologies, the ubiquity of MBHs in nearby luminous galaxies can arise even if only a small fraction of halos harbor seed 

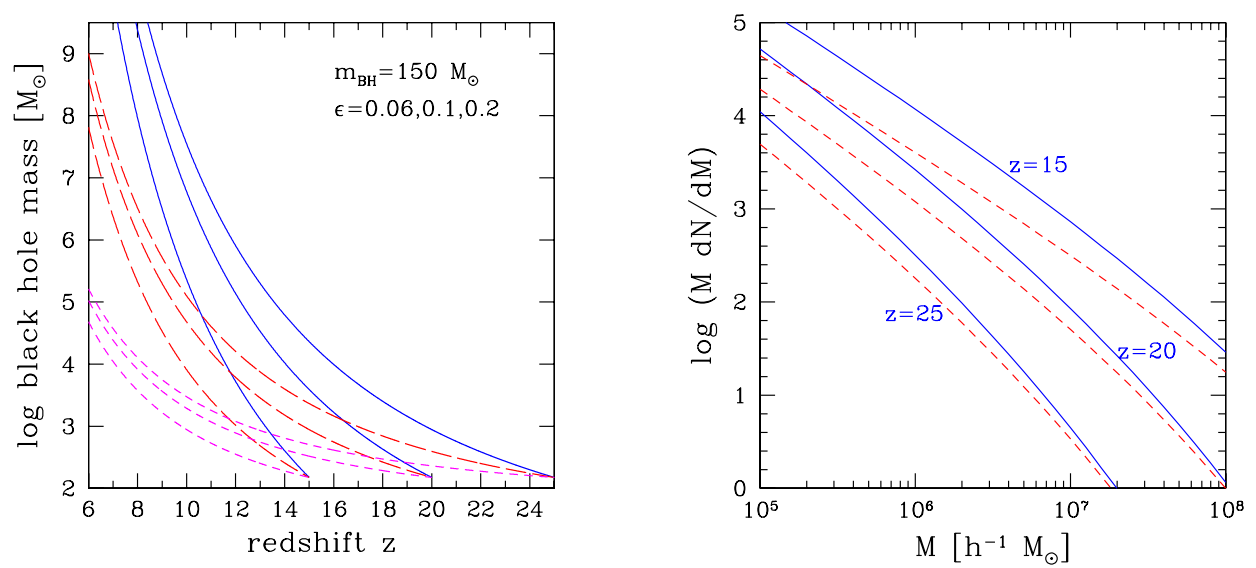

Figure 1. Left: Growth of MBHs from early epochs down to $z=6$, the redshift of the most distant SDSS quasars. The three sets of curves assume Eddington-limited accretion with radiative efficiency $\epsilon=0.06$ (solid lines), 0.1 (long-dashed lines), and 0.2 (short-dashed lines). Gas accretion starts at $z=15,20,25$ onto a seed black of mass $m_{\mathrm{BH}}=150 \mathrm{M}_{\odot}$. Right: Mass function of progenitor dark matter halos of mass $M$ at $z=15,20,25$ which, by the later time $z_{0}$, will have merged into a larger host of mass $M_{0}$. Solid curves: $z_{0}=0.8, M_{0}=10^{12} h^{-1} \mathrm{M}_{\odot}$ ("Milky Way" halo). Dashed curves: $z_{0}=3.5, M_{0}=2 \times 10^{11} h^{-1} \mathrm{M}_{\odot}$ (older "bulge"). If one seed hole formed in each $\sim 10^{6} \mathrm{M}_{\odot}$ minihalo collapsing at $z \sim 20$ (and triple hole interactions and binary coalescences were neglected), several thousands relic seed black holes and their descendants would be orbiting within the halos of present-day galaxies. (From Madau \& Rees 2001.)

holes at very high redshift (Menou, Haiman \& Narayanan 2001). The origin and nature of this seed population remain uncertain. Numerical simulations of the fragmentation of primordial molecular clouds in hierarchical cosmologies all show the formation of Jeans unstable clumps with masses exceeding a few hundred solar masses, with the implication that the resulting initial mass function is likely to be biased to very massive "Population III" stars (Abel, Bryan \& Norman 2002; Bromm, Coppi \& Larson 2002). Barring any fine tuning of the IMF, intermediate-mass black holes - with masses above the $4-18 \mathrm{M}_{\odot}$ range of known stellar-mass holes - may then be the inevitable end-product of the first episode of pregalactic star formation.

\section{Massive black holes as Population III remnants}

The first stars in the universe formed out of metal-free gas, in dark matter "minihalos" collapsing from the high- $\sigma$ peaks of the primordial density field at redshift $z>15$ or so. Gas condensation in the first baryonic objects was possible through the formation of $\mathrm{H}_{2}$ molecules, which cool via roto-vibrational transitions down to temperatures of a couple hundred Kelvin. At zero metallicity mass loss through radiatively-driven stellar winds or nuclear-powered stellar pulsations is expected to be negligible, and Pop III stars will likely die losing only a small fraction of their mass (except for $100<m_{*}<140 \mathrm{M}_{\odot}$ ). Nonrotating very massive stars in the mass window $140 \lesssim m_{*} \lesssim 260 \mathrm{M}_{\odot}$ will disappear as pair-instability supernovae (Bond, Arnett \& Carr 1984), leaving no compact remnants 
and polluting the universe with the first heavy elements (Oh et al. 2001). $\dagger$ Stars with $40<m_{*}<140 \mathrm{M}_{\odot}$ and $m_{*}>260 \mathrm{M}_{\odot}$ are predicted instead to collapse to black holes with masses exceeding half of the initial stellar mass (Heger \& Woosley 2002). Since they form in high- $\sigma$ rare density peaks, relic seed black holes are expected to cluster in the bulges of present-day galaxies as they become incorporated through a series of mergers into larger and larger systems (see Figure 1). The presence of a small cluster of Population III holes in galaxy nuclei may have several interesting consequences associated with tidal captures of ordinary stars (likely followed by disruption), capture by the central supermassive hole, and gravitational wave radiation from such coalescences. Accreting pregalactic seed holes may be detectable as ultra-luminous, off-nuclear X-ray sources (Madau \& Rees 2001).

\section{The first miniquasars}

Physical conditions in the central potential wells of young and gas-rich protogalaxies may have been propitious for black hole gas accretion. The presence of accreting black holes powering Eddington-limited miniquasars at such crucial formative stages in the evolution of the universe may then present a challenge to models of the epoch of first light and of the thermal and ionization early history of the intergalactic medium (IGM), as reheating from the first stars and their remnants likely played a key role in structuring the IGM and in regulating gas cooling and star formation in pregalactic objects. Energetic photons from miniquasars may make the low-density IGM warm and weakly ionized prior to the epoch of reionization breakthrough (Madau et al. 2004; Ricotti, Ostriker \& Gnedin 2005). X-ray radiation may boost the free-electron fraction and catalyze the formation of $\mathrm{H}_{2}$ molecules in dense regions, counteracting their destruction by UV Lyman-Werner radiation (Haiman, Abel \& Rees 2000; Machacek, Bryan \& Abel 2003). Or it may furnish an entropy floor to the entire IGM, preventing gas contraction and therefore impeding rather than enhancing $\mathrm{H}_{2}$ formation (Oh \& Haiman 2003). Photoionization heating may evaporate back into the IGM some of the gas already incorporated into halos with virial temperatures below a few thousand Kelvin (Barkana \& Loeb 1999). The detailed consequences of all these effects are poorly understood. In the absence of a UV photodissociating flux and of ionizing X-ray radiation, 3D simulations show that the fraction of cold, dense gas available for star formation or accretion onto seed holes exceeds $20 \%$ for halos more massive than $10^{6} \mathrm{M}_{\odot}$ (Figure 2; Machacek et al. 2003; Kuhlen $\&$ Madau 2005). Since a zero-metallicity progenitor star in the range $40<m_{*}<500 \mathrm{M}_{\odot}$ emits about 80,000 photons above 1 ryd per stellar baryon (Schaerer 2002), the ensuing ionization front may overrun the host halo, photoevaporating most of the surrounding gas. Black hole remnants of the first stars that created $\mathrm{H}$ II regions are then unlikely to accrete significant mass until new cold material is made available through the hierarchical merging of many gaseous subunits.

High-resolution hydrodynamics simulations of early structure formation in $\Lambda$ CDM cosmologies are a powerful tool to track in detail the thermal and ionization history of a clumpy IGM and guide studies of early reheating. In Kuhlen \& Madau (2005) we used a modified version of ENZO, a grid-based hybrid (hydro+N-body) code developed by Bryan \& Norman (see http://cosmos.ucsd.edu/enzo/) to solve the cosmological hydrodynamics equations and simulate the effect of a miniquasar turning on at very high redshift. The

$\dagger$ Since primordial metal enrichment was intrinsically a local process, pair-instability $\mathrm{SNe}$ may occur in pockets of metal-free gas over a broad range of redshifts. The peak luminosities of typical pair-instability SNe are only slightly greater than those of Type Ia, but they remain bright much longer ( 1 year) and have hydrogen lines (Scannapieco et al. 2005). 

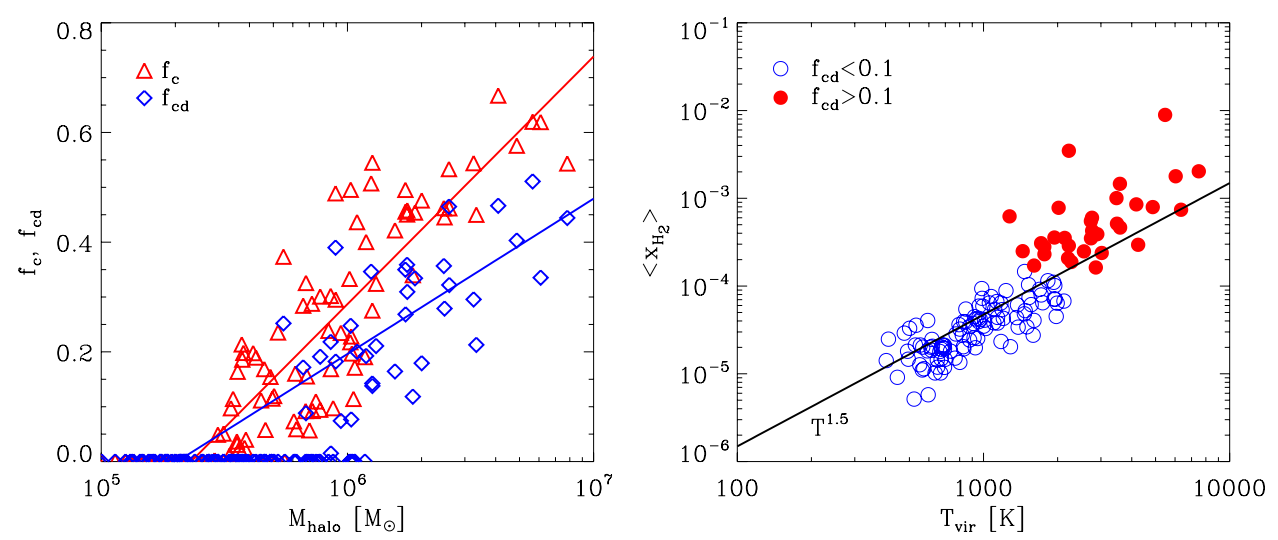

Figure 2. Left: Fraction of cold and cold+dense gas within the virial radius of all halos identified at $z=17.5$ with $T_{\mathrm{vir}}>400 \mathrm{~K}$, as a function of halo mass. Triangles: $f_{c}$, fraction of halo gas with $T<0.5 T_{\text {vir }}$ and $\delta>1000$ that cooled via roto-vibrational transitions of $\mathrm{H}_{2}$. Diamonds: $f_{\text {cd }}$, fraction of gas with $T<0.5 T_{\text {vir }}$ and $\rho>10^{19} \mathrm{M}_{\odot} \mathrm{Mpc}^{-3}$ that is available for star formation. The straight lines represent mean regression analyses of $f_{c}$ and $f_{\text {cd }}$ with the logarithm of halo mass. Right: Mass-weighted mean $\mathrm{H}_{2}$ fraction as a function of virial temperature for all halos at $z=17.5$ with $T_{\text {vir }}>400 \mathrm{~K}$ and $f_{\text {cd }}<0.1$ (empty circles) or $f_{\text {cd }}>0.1$ (filled circles). The straight line marks the scaling of the temperature-dependent asymptotic molecular fraction. (From Kuhlen \& Madau 2005.)

simulation follows the non-equilibrium chemistry of the dominant nine species $\left(\mathrm{H}, \mathrm{H}^{+}\right.$, $\mathrm{H}^{-}$, e, $\mathrm{He}, \mathrm{He}^{+}, \mathrm{He}^{++}, \mathrm{H}_{2}$, and $\mathrm{H}_{2}^{+}$) in primordial gas, and includes radiative losses from atomic and molecular line cooling. At $z=21$, a miniquasar powered by a $150 \mathrm{M}_{\odot}$ black hole accreting at the Eddington rate is turned on in the protogalactic halo. The miniquasar shines for a Salpeter time (i.e. down to $z=17.5$ ) and is a copious source of soft X-ray photons, which permeate the IGM more uniformly than possible with extreme ultraviolet $(\mathrm{EUV}, \geqslant 13.6 \mathrm{eV})$ radiation. After one Salpeter timescale, the miniquasar has heated up the simulation box to a volume-averaged temperature of $2800 \mathrm{~K}$. The mean electron and $\mathrm{H}_{2}$ fractions are now 0.03 and $4 \times 10^{-5}$ : the latter is 20 times larger than the primordial value, and will delay the buildup of a uniform UV photodissociating background. The net effect of the X-rays is to reduce gas clumping in the IGM by as much as a factor of 3 . While the suppression of baryonic infall and the photoevaporation of some halo gas lower the gas mass fraction at overdensities $\delta$ in the range 20-2000, enhanced molecular cooling increases the amount of dense material at $\delta>2000$. In many halos within the proximity of our miniquasar the $\mathrm{H}_{2}$-boosting effect of $\mathrm{X}$-rays is too weak to overcome heating, and the cold and dense gas mass actually decreases. There is little evidence for an entropy floor preventing gas contraction and $\mathrm{H}_{2}$ formation: instead, molecular cooling can affect the dynamics of baryonic material before it has fallen into the potential well of dark matter halos and virialized. Overall, the radiative feedback from X-rays enhances gas cooling in lower- $\sigma$ peaks that are far away from the initial site of star formation, thus decreasing the clustering bias of the early pregalactic population, but does not appear to dramatically reverse or promote the collapse of pregalactic clouds as a whole. 


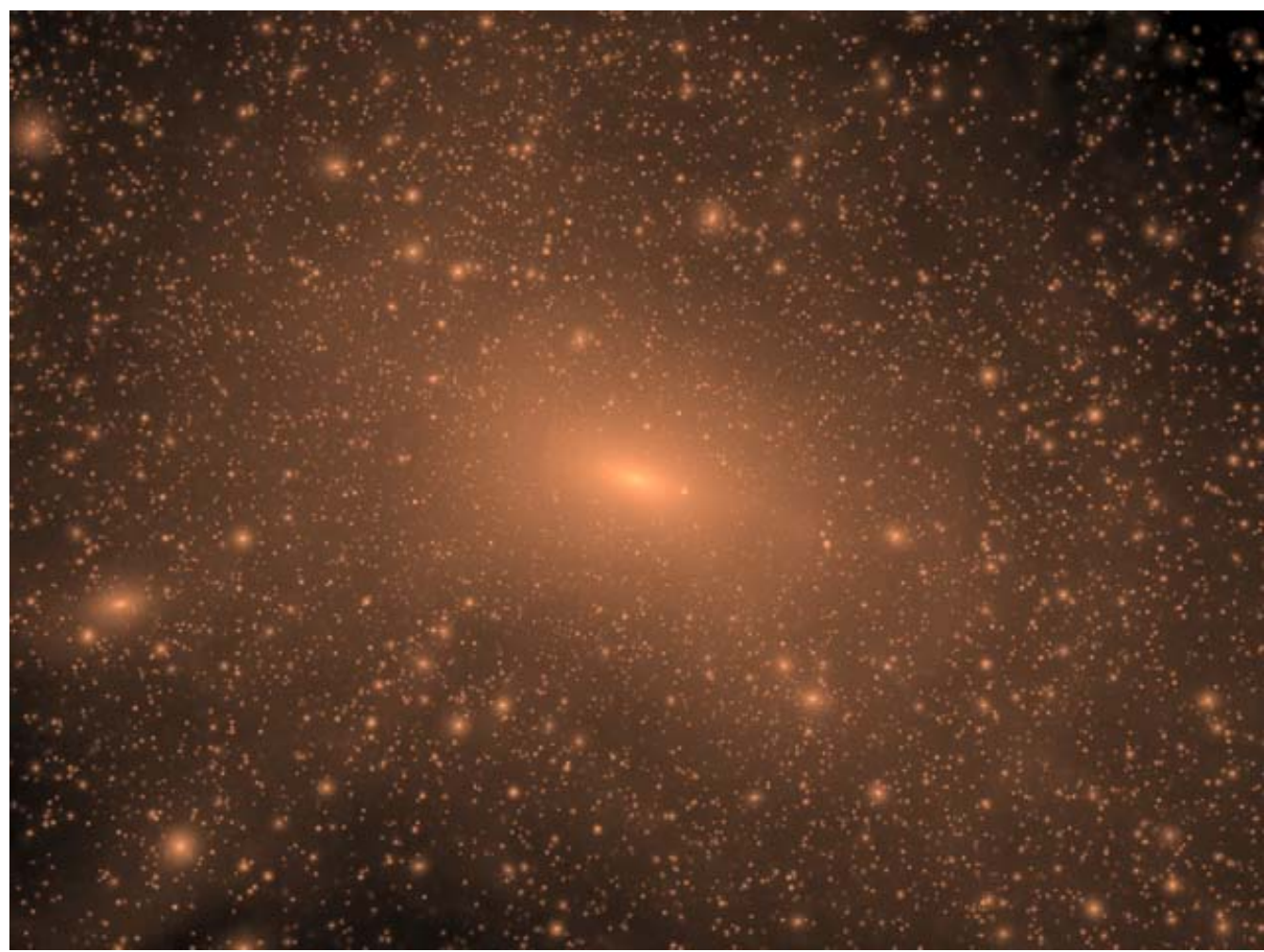

Figure 3. Projected dark matter density-squared map of our simulated Milky Way-size halo ("Via Lactea") at the present epoch. The image covers an area of $800 \times 600 \mathrm{kpc}$, and the projection goes through a $600 \mathrm{kpc}$-deep cuboid containing a total of 110 million particles. The logarithmic color scale covers 20 decades in density-square. (From Diemand, Kuhlen \& Madau 2006.)

\section{Near-field cosmology and dark satellites}

Despite much recent progress in our understanding of the formation of early cosmic structure and the high-redshift universe, many fundamental questions related to the astrochemistry of primordial gas and the formation and evolution of halo+MBH systems remain only partially answered. N-body+hydrodynamical simulations are unable to predict, for example, the efficiency with which the first gravitationally collapsed objects lit up the universe at cosmic dawn, and treat the effects of the energy input from the earliest generations of sources on later ones only in an approximate way. The Wilkinson Microwave Anisotropy Probe 3-year data require the universe to be fully reionized by redshift $z_{\text {ion }}=11 \pm 2.5$ (Spergel et al. 2006), an indication that significant star-formation activity started at early cosmic times. We infer that Population III massive stars and perhaps miniquasars must have been shining when the universe was less than $350 \mathrm{Myr}$ old, but we remain uncertain about the nature of their host galaxies and the impact they had on their environment and on the formation and structure of more massive systems.

While the above cosmological puzzles can be tackled directly by studying distant objects, it has recently become clear that many of today's "observables" within the Milky Way and nearby galaxies relate to events occurring at very high redshifts, during and soon after the epoch of reionization (see Moore et al. 2006 and references therein). In 

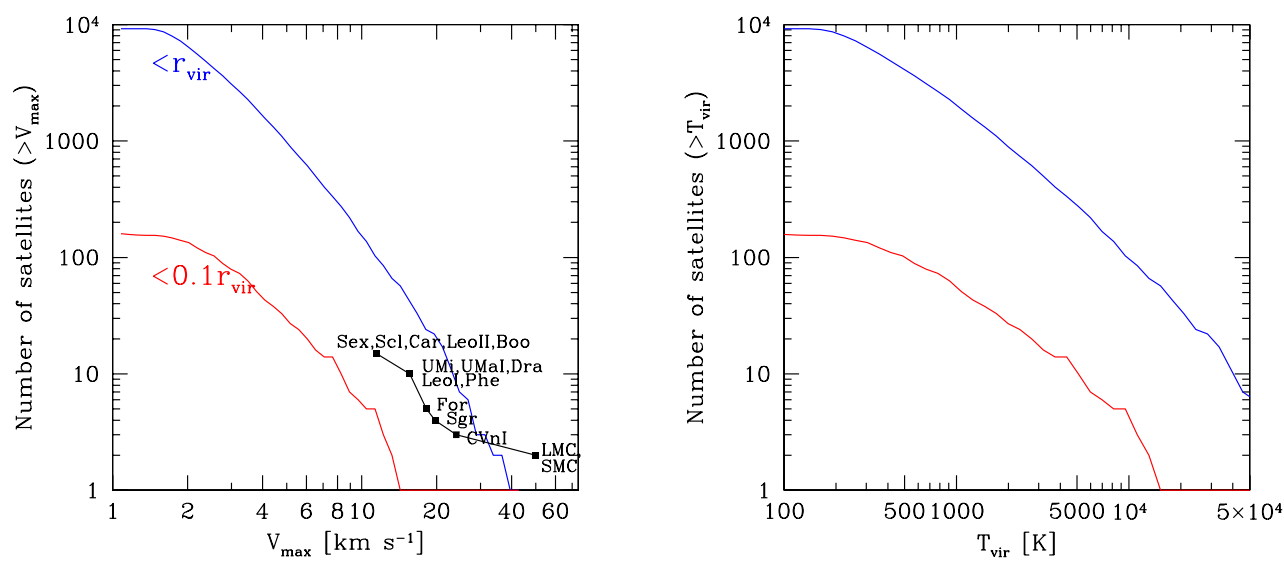

Figure 4. Left: Cumulative peak circular velocity function for all subhalos within Via Lactea's $r_{\text {vir }}$ (upper curve) and for the subpopulation within the inner $0.1 r_{\text {vir }}$ (lower curve). Solid line with points: observed number of dwarf galaxy satellites around the Milky Way. Right: Same plotted versus virial temperature $T_{\mathrm{vir}}$.

this sense, galaxies in the Local Group can provide a crucial diagnostic link to the physical processes that govern structure formation and evolution in the early universe, an approach termed "near-field cosmology". It is now well established that the hierarchical mergers that form the halos surrounding galaxies are rather inefficient, leaving substantial amounts of stripped halo cores or "subhalos" orbiting within these systems. Small halos collapse at high redshift when the universe is very dense, so their central densities are correspondingly high. When these merge into larger hosts, their high densities allow them to resist the strong tidal forces that acts to destroy them. Gravitational interactions appear to unbind most of the mass associated with the merged progenitors, but a significant fraction of these small halos survives as distinct substructure.

In order to recognize the basic building blocks of galaxies in the near-field, we have recently completed "Via Lactea", the most detailed simulation of Milky Way CDM substructure to date (Diemand, Kuhlen \& Madau 2006). The simulation resolves a Milky Way-size halo with 85 million particles within its virial radius $r_{\text {vir }}$, and was run for 320,000 cpu-hours on NASA's SGI Altix supercomputer Columbia. Figure 3 shows a projected dark matter density squared map of a $800 \times 600 \mathrm{kpc}$ region of this simulation at the current epoch. The high resolution region was sampled with 234 million particles of mass $2.1 \times 10^{4} \mathrm{M}_{\odot}$, evolved with a force resolution of $90 \mathrm{pc}$, and centered on an isolated halo that had no major merger after $z=1.7$, making it a suitable host for a Milky Way-like disk galaxy.

Approximately 10,000 surviving satellite halos can be identified at the present epoch: this is more than an order of magnitude larger than found in previous $\Lambda$ CDM simulations. Their cumulative mass function is well-fit by $N\left(>M_{\text {sub }}\right) \propto M_{\text {sub }}^{-1}$ down to $M_{\text {sub }}=4 \times 10^{6} \mathrm{M}_{\odot}$. Sub-substructure is apparent in all the larger satellites, and a few dark matter lumps are now resolved even in the solar vicinity. In Via Lactea, the number of dark satellites with peak circular velocities above $5 \mathrm{~km} \mathrm{~s}^{-1}\left(10 \mathrm{~km} \mathrm{~s}^{-1}\right)$ exceeds 800 (120). As shown in Figure 4, such finding appears to exacerbate the so-called "missing satellite problem", the large mismatch between the twenty or so dwarf satellite galaxies observed around the Milky Way and the predicted large number of CDM subhalos 


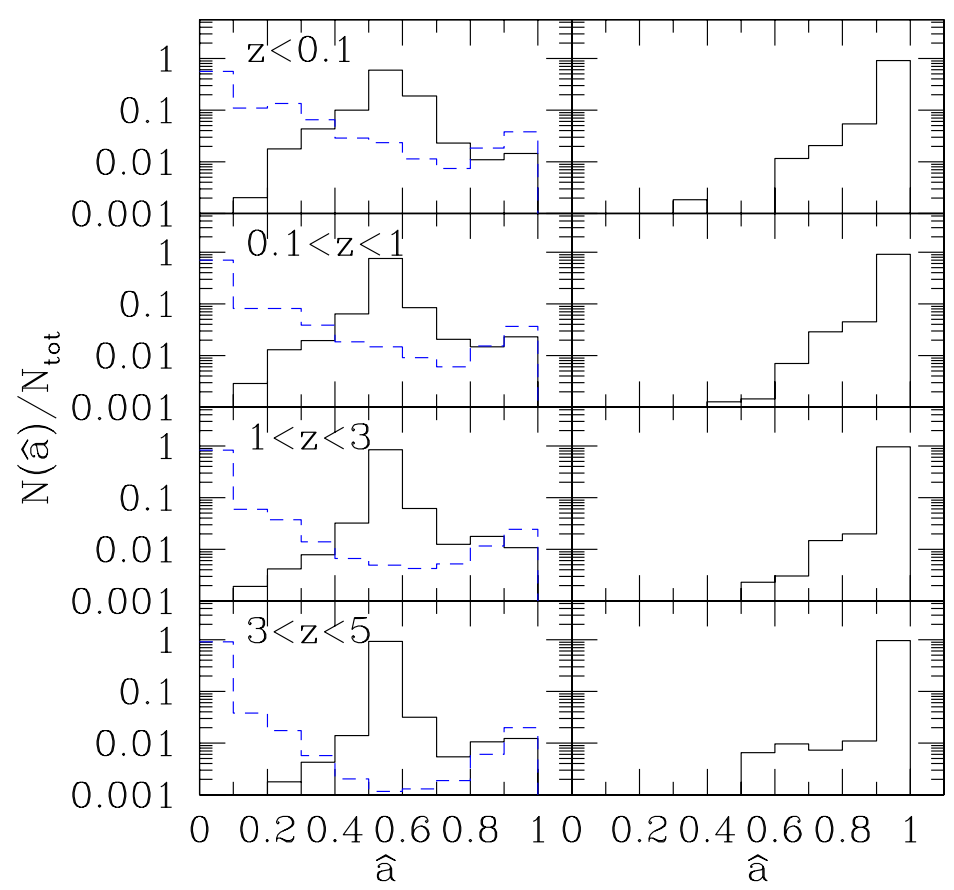

Figure 5. Distribution of MBH spins in different redshift intervals. Left panel: effect of black hole binary coalescences only. Solid histogram: seed holes are born with $\hat{a} \equiv a / m_{\mathrm{BH}}=0.6$. Dashed histogram: seed holes are born non-spinning. Right panel: spin distribution from binary coalescences and gas accretion. Seed holes are born with $\hat{a}=0.6$, and are efficiently spun up by accretion via a thin disk.

(Moore et al. 1999; Klypin et al. 1999). Solutions involving feedback mechanisms that make halo substructure very inefficient in forming stars offer a possible way out (e.g. Bullock, Kravtsov \& Weinberg 2000; Kravtsov, Gnedin \& Klypin 2004; Moore et al. 2006). In this case seed black holes may not have grown efficiently in small minihalos just above the cosmological Jeans mass, and gas accretion may have had to await the buildup of more massive galaxies (with virial temperatures above the threshold for atomic cooling).

\section{MBH spins}

As discussed in the Introduction, growing very large MBHs at high redshift from small seeds requires low accretion efficiencies, or, equivalently, modest black hole spins. The spin of a $\mathrm{MBH}$ is also expected to have implications for the direction of jets in active nuclei and to determine the innermost flow pattern of gas accreting onto Kerr holes (Bardeen \& Petterson 1975). The coalescence of two spinning black holes in a radio galaxy may cause a sudden reorientation of the jet direction, perhaps leading to the so-called "winged" or "X-type" radio sources (Merritt \& Ekers 2002). MBH spins are determined by the competition between a number of physical processes. Black holes forming from the gravitational collapse of very massive stars endowed with rotation will in general be born with non-zero spin (e.g. Fryer, Woosley \& Heger 2002). An initially 
non-rotating hole that increases its mass by (say) $50 \%$ by swallowing material from an accretion disk may be spun up to $a / m_{\mathrm{BH}}=0.84$. While the coalescence of two nonspinning black holes of comparable mass will immediately drive the spin parameter of the merged hole to $a / m_{\mathrm{BH}} \gtrsim 0.8$ (e.g. Gammie, Shapiro \& McKinney 2004), the capture of smaller companions in randomly-oriented orbits may spin down a Kerr hole instead (Hughes \& Blandford 2003).

In Volonteri et al. (2005) we made an attempt at estimating the distribution of MBH spins and its evolution with cosmic time in the context of hierarchical structure formation theories, following the combined effects of black hole-black hole coalescences and accretion from a gaseous thin disk on the magnitude and orientation of MBH spins. Binary coalescences appear to cause no significant systematic spin-up or spin-down of MBHs: because of the relatively flat distribution of $\mathrm{MBH}$ binary mass ratios in hierarchical models, the holes random-walk around the spin parameter they are endowed with at birth, and the spin distribution retains significant memory of the initial rotation of "seed" holes. It is accretion, not binary coalescences, that dominates the spin evolution of MBHs (Fig. 5). Accretion can lead to efficient spin-up of MBHs even if the angular momentum of the inflowing material varies in time, provided the fractional change of mass during each accretion episode of a growing black hole is large. In this case, for a thin accretion disk, the hole is aligned with the outer disk on a timescale that is much shorter than the Salpeter time (Natarajan \& Pringle 1998), leading to accretion via prograde equatorial orbits: most of the mass accreted by the hole acts to spin it up, even if the orientation of the spin axis changes in time. Under the combined effects of accretion and binary coalescences, we found that the spin distribution is heavily skewed towards fast-rotating Kerr holes, is already in place at early epochs, and does not change significantly below redshift 5. One way to avoid rapid rotation and produce slowly rotating fast-growing holes is to assume "chaotic feeding" in which small amounts of material, with $\Delta m \ll m_{\mathrm{BH}}$, are swallowed by the hole in successive accretion episodes with random orientations (e.g., Moderski and Sikora 1996; Volonteri et al. 2005; King \& Pringle 2006).

\section{Acknowledgements}

I would like to thank all my collaborators for their contributions to the ideas presented here. Support for this work was provided by NASA grant NNG04GK85G.

\section{References}

Abel T., Bryan G. \& Norman, M. L. 2002, Science, 295, 93

Bardeen J. M. \& Petterson J. A. 1975, ApJ, 195, L65

Barkana R. \& Loeb A. 1999, ApJ, 523, 54

Bond J. R., Arnett W. D. \& Carr B. J. 1984, ApJ, 280, 825

Bromm V., Coppi P. S. \& Larson R. B. 2002, ApJ, 564, 23

Bullock J. S., Kravtsov A. V. \& Weinberg D. H. 2000, ApJ, 539, 517

Diemand J., Kuhlen M. \& Madau P. 2006, ApJ, in press (astro-ph/0611370)

Ferrarese L. \& Merritt D. 2000, ApJ, 539, L9

Fryer C. L., Woosley S. E. \& Heger A. 2001, ApJ, 550, 372

Gammie C. F., Shapiro S. L. \& McKinney J. C. 2004, ApJ, 602, 312

Gebhardt K. et al. 2000, ApJ, 543, L5

Haiman Z., Abel T. \& Rees M. J. 2000, ApJ, 534, 11

Heger A. \& Woosley S. E. 2002, ApJ, 567, 532

Hughes S. A. \& Blandford R. D. 2003, ApJ, 585, L101

King A. R. \& Pringle J. E. 2006, MNRAS, 373, L90 
Klypin A. A., Kravtsov A. V., Valenzuela O. \& Prada F. 1999, ApJ, 522, 82

Kravtsov A. V., Gnedin O. Y. \& Klypin A. A. 2004, ApJ, 609, 482

Kuhlen M. \& Madau P. 2005, MNRAS, 363, 1069

Menou K., Haiman Z. \& Narayanan V. K. 2001, ApJ, 558, 535

Machacek M. M., Bryan G. L. \& Abel T. 2003, MNRAS, 338, 273

Madau P. \& Rees M. J. 2001, ApJ, 551, L27

Madau P., Rees M. J., Volonteri M., Haardt F. \& Oh S. P. 2004, ApJ, 606, 484

Merritt D. \& Ekers R. D. 2002, Science, 297, 1310

Moderski R. \& Sikora M. 1996, A\&AS, 120, 591

Moore B., Diemand J., Madau P., Zemp M. \& Stadel J. 2006, MNRAS, 368, 563

Moore B., Quinn T., Governato F., Stadel J. \& Lake G. 1999, MNRAS, 310, 1147

Natarajan P. \& Pringle J. E. 1998, ApJ, 506, L97

Oh S. P. \& Haiman Z. 2003, MNRAS, 346, 456

Oh S. P., Nollett K. M., Madau P. \& Wasserburg G. J. 2001, ApJ, 562, L1

Ricotti M., Ostriker J. P. \& Gnedin N. Y. 2005, MNRAS, 357, 207

Scannapieco E., Madau P., Woosley S., Heger A. \& Ferrara A. 2005, ApJ, 633, 1031

Schaerer D. 2002, A\&A, 382, 28

Spergel D. N., et al. 2006, ApJ, submitted (astro-ph/0603449)

Tegmark M., Silk J., Rees M. J., Blanchard A., Abel T. \& Palla F. 1997, ApJ, 474, 1

Volonteri M., Madau P., Quataert E. \& Rees M. J. 2005, ApJ, 620, 69

Volonteri M. \& Rees M. J. 2005, ApJ, 633, 624

Mitchell Begelman: Comment: If your are able to surround a small seed black hole with a sufficiently optically thick envelope that is much more massive than the seed black hole then the relevant Eddington limit is actually the Eddington limit for that envelope, not for the mass of the black hole. You could in principle accrete about 100 time the Eddington limit or even more, up to about $10^{4}-10^{5}$ solar masses.

Piero Madau: Thanks for pointing this out. Such a supercritical accretion phase would help in relaxing some of the constraints associated with the early growth of billion solar mass holes by redshift 6 .

Martin Rees: Just a less radical comment. The "classic" efficiency arguments based on the binding energy of the innermost stable orbit are of course not strictly valid when the accretion is at the critical rate - the disc is then thick and the thickness is of the order of the Schwarzschild radius. As has been known since the 1980s, even with the Schwarzschild case the efficiency can be either lower or higher than the standard thin-disc value.

PIERo MADAU: Thanks Martin. I thought about thick radiation-supported tori - I was working on those configurations long time ago with my $\mathrm{PhD}$ supervisor, Marek Abramowicz. We have run a few models and they do not seem to help much with the "spin problem": as the radiation efficiency drops, the holes accrete material with angular momentum and spin up rapidly.

Günther HAsinger: The Supernova that produces the black hole in the first place could wipe out its birth halo because of the high explosion velocity. Could this be the reason for subhalos below the atomic cooling mass to be dark?

PIERo MAdau: Population III very massive stars above 260 solar masses are predicted to collapse to black holes without ejecting material. In general, feedback from supernova explosion is a plausible mechanism for suppressing further star formation in young small 
galaxies. Of course, and contrary to reionization from the "outside", it must be at work in every minihalo at high redshifts.

FUKUn LIU: One comment on the impact of recoil velocity on the formation of massive black holes in dwarf galaxies. In your simulations, you have assumed that black hole binary coalesces instantly when two galaxies merge. However, we know that dynamical friction becomes inefficient before gravitational wave radiation starts to dominate the loss of binary orbital angular momentum, so we do not know how black hole binaries become harder. Up to now the observational evidence for black hole binary merges, e.g. double radio galaxies proposed by us (Liu et al. 2003, MNRAS) or X-shaped radio sources suggested by Merritt \& Ekers (2002, Science), are relevant for massive galaxies. Therefore, it is quite possible that two black holes in dwarf galaxies do not coalesce but grow separately until the next galaxy merger occurs and the host galaxy becomes massive.

PIERO MADAU: I agree. In this work we just simply assumed that two black holes coalesce on short timescales in order to investigate the effect of $\mathrm{MBH}$ coalescences on the spin distribution. 